\title{
Treatment of Mild to Moderate Hallux Valgus, Percutaneous Osteotomy versus Distal Chevron Osteotomy
}

\author{
Ahmed Mohamed Ahmed Othman ${ }^{*}$, Islam Hassan Ali Hegazy ${ }^{2}$ \\ ${ }^{1}$ Department of Orthopedic Surgery, Faculty of Medicine, El-Minia University, El-Minia, Egypt \\ ${ }^{2}$ Department of Orthopedic Surgery, Faculty of Medicine, Benha University, Benha, Egypt \\ Email: "osman682012@yahoo.com
}

Received 1 May 2016; accepted 10 June 2016; published 13 June 2016

Copyright (C) 2016 by authors and Scientific Research Publishing Inc.

This work is licensed under the Creative Commons Attribution International License (CC BY). http://creativecommons.org/licenses/by/4.0/

c) (7) Open Access

\begin{abstract}
Objective: The aim of this study was to compare two different surgical techniques for treating mild to moderate cases of hallux valgus. Methods: Forty-one patients (58 feet) with mild to moderate hallux valgus were treated by either percutaneous technique $(n=24)$ patients $(33$ feet $)$ or by distal chevron osteotomy $(n=17)(25$ feet). Results: In the percutaneous group, after a mean follow up of 49.36 months, the mean correction of hallux valgus angle (HV) was $26.69^{\circ}$; in the intermetatarsal angle, average correction was $9.45^{\circ}$. The mean improvement of American Orthopedic Foot and Ankle Score (AOFAS) was 46.45 points. In the chevron osteotomy group, the mean follow up of 51.56 months, the mean correction of hallux valgus angle was $26.78^{\circ}$; in the intermetatarsal angle, average correction was $9^{\circ}$. The mean improvement of AOFAS was 44.76 points. Conclusion: Both techniques gave satisfactory results. However, the percutaneous technique is safer than the distal chevron osteotomy.
\end{abstract}

\section{Keywords}

Hallux Valgus, Percutaneous Technique, Chevron Osteotomy, AOFAS

\section{Introduction}

Hallux valgus (HV) is a common forefoot deformity. It is described as a valgus angulation of the first metatarsophalangeal (MTP) joint of the great toe. The condition is frequently painful and may lead to limitation of physical activities. Sometimes it causes psychological distress for patients. The deformity includes not only the

${ }^{*}$ Corresponding author.

How to cite this paper: Othman, A.M.A. and Hegazy, I.H.A. (2016) Treatment of Mild to Moderate Hallux Valgus, Percutaneous Osteotomy versus Distal Chevron Osteotomy. Open Journal of Orthopedics, 6, 150-157.

http://dx.doi.org/10.4236/ojo.2016.66023 
outward lateral deviation of the great toe but also a medial deviation of the first metatarsal bone [1].

Several surgical procedures have been described for treatment of HV deformity including soft tissue procedures, distal metatarsal osteotomies, proximal metatarsal osteotomies, scarf osteotomy etc.; however, none of them is universally suitable for all patients.

Surgical correction of hallux valgus aims at morphologic and functional rebalancing of the first ray. Many techniques are reported to reach these goals, each of them with different indications depending on the pathoanatomy of the deformity requiring treatment. Many authors have reported their experience using various types of distal metatarsal osteotomies characterized by different indications, approaches, designs, and methods of fixation [2].

\section{There Are Four Radiological Parameters Which Should Be Documented before the Start of Treatment [1]}

1) The hallux valgus angle (HVA) is the intersection of the longitudinal axes of the proximal phalanx and the first MT. A normal HV angle is less than $15^{\circ}$.

2) The intermetatarsal angle (IMA) or sometimes referred as M1-M2 angle, is the intersection of the longitudinal axes of the first and second metatarsals. Less than $9^{\circ}$ is considered normal.

3) The distal metatarsal articular angle (DMAA) describes the relationship between the distal articular surface and the long axis of the first MT (M1). A normal angle is less than $10^{\circ}$.

Accordingly, the deformity is classified as mild moderate and severe. Mild deformity is defined by an IMA greater than $13^{\circ}$ but the HVA is less than $40^{\circ}$. Moderate deformity is characterized by an IMA of greater than $20^{\circ}$ and HVA greater than $40^{\circ}$. Severe deformity Moderate deformity is characterized by an IMA of greater than $20^{\circ}$ and HVA greater than $40^{\circ}$.

The ideal surgical procedure is supposed to correct hallux valgus angle (HVA) and the inter metatarsal angle (IMA), restore joint congruity, eliminate pain, and preserve range of motion [3].

There is much controversy concerning forefoot surgery. Certain concepts such as conventional procedures, minimally invasive surgery, or percutaneous surgery are promoted because of their specific advantages including rapid recovery and compatibility with a short hospital stay or even outpatient surgery [4].

The use of minimal invasive surgical procedures is becoming more popular. Percutaneous techniques are currently used in many surgical procedures on the soft tissues and bones of the foot [5] [6].

The chevron osteotomy is a medial eminence resection, distal MT osteotomy, and medial capsulorrhaphy. It is indicated in cases include a $\mathrm{HV}$ angle less than $30^{\circ}$, a 1-2 IM angle less than $13^{\circ}$, subluxation of the MTP joint, and a congruous MTP joint if the distal metatarsal articular angle (DMAA) is less than $15^{\circ}$. However, chevron osteotomy does not correct pronation and only partially corrects the position of sesamoids [1].

\section{Patients and Methods (Table 1)}

Between January 2009 and September 2014, forty one patients (58 feet) suffering from mild to moderate hallux valgus were randomly assigned into two groups according to the method of treatment. An informed consent was obtained from all patients included in the study. The confidentiality of information collected has been preserved.

The patients were divided into 2 groups. The first group included 24 patients (33 feet) there were 9 bilateral and 15 unilateral. This group was treated by percutaneous osteotomy. There were 22 females and 2 males. The

\begin{tabular}{cccc} 
Table 1. Pre-operative data. & & & \\
\hline & Percutaneous & Chevron & P value \\
\hline Age & 40.57 & 39.2 & 0.87943 \\
HV angle & 33.24 & 33.3 & 0.65743 \\
IM angle & 17.03 & 16.96 & 0.98742 \\
Tibial sesamoid position & 8.51 & 8.4 & 0.78643 \\
DMAA & 19.84 & 19.4 & 0.69531 \\
AOFAS & 43.15 & 42.04 & 0.78645 \\
\hline
\end{tabular}


age ranged between 29 and 55 years with an average of 40.57 years old. The AOFAS score ranged between 33 and 48 with an average of 43.15 , the hallux valgus angle (HV) ranged between $20^{\circ}$ and $40^{\circ}$ with an average of $33.24^{\circ}$. The intermetatarsal angle (M1-M2 angle) ranged between $15^{\circ}$ and $19^{\circ}$ with an average of $17.03^{\circ}$. The distal metatarsal articular angle (DMAA) ranged between $16^{\circ}$ and $24^{\circ}$ with an average of $19.84^{\circ}$. The tibial sesamoid position ranged between $5 \mathrm{~mm}$ and $11 \mathrm{~mm}$ with an average of $8.51 \mathrm{~mm}$.

The second group included 17 patients (25 feet), 8 bilateral and 9 unilateral were treated by distal chevron osteotomy. There were 16 females and 1 male. The age ranged between 23 and 51 years with an average of 39.2 years. The AOFAS ranged between 32 and 46 with an average of 42.04, the HV angle ranged between 20 degrees and 40 degrees with an average of $33.72^{\circ}$. The M1-M2 angle ranged between 14 and 18 degrees with an average of $16.96^{\circ}$. The DMAA ranged between $16^{\circ}$ and $23^{\circ}$ with an average of $19.4^{\circ}$. The tibial sesamoid position ranged between $4 \mathrm{~mm}$ and $12 \mathrm{~mm}$ with an average of $8.4 \mathrm{~mm}$.

\subsection{Inclusion Criteria}

Painful mild to moderate hallux valgus deformity not responding to conservative measures.

\subsection{Exclusion Criteria}

Recurrent hallux valgus deformity after previous surgery. Severe hallux valgus (HVA above $40^{\circ}$, IMA above $20^{\circ}$ ). Marked degenerative changes of the first metatarsophalangeal joint (hallux valgus et rigidus) and associated deformity or abnormality in the foot and ankle.

Cases with bilateral affection were treated by similar technique i.e. if we started treatment of one patient with chevron or percutaneous technique for one foot, the other foot is to be operated by the same type of surgery.

\subsection{Preoperative Assessment}

The entire foot is thoroughly examined clinically while the patient is standing, sitting, and lying supine. The forefoot is examined for corns, calluses, warts, interdigital neuromas, bunionettes, hammer toes, and claw toes. Finally, the midfoot and hindfoot must be examined carefully before making treatment recommendations for forefoot surgery. Also, the foot is examined for vascularity and sensibility.

\subsection{Radiological Evaluation}

Weight bearing dorso-plantar and lateral radiographs of the foot were obtained preoperatively and at the time of follow-up. The hallux valgus angle (HVA), IMA, distal metatarsal articular angle (DMAA), congruency of the first MTP joint, and the position of the medial sesamoid were measured on weight bearing dorso-plantar radiographs preoperatively and at final follow-up using a digital picture archiving and communication system (PACS) image system.

Surgical technique: All surgeries were done under ultrasound guided regional anesthesia and tourniquet applied to the upper thigh.

\subsection{The Percutaneous Technique [7]}

A $1 \mathrm{~cm}$ skin incision centered over the medial aspect of the first metatarsal neck midway between the dorsal and plantar aspects of the first metatarsal bone cutting the periosteum down to bone. A 1.8 or 2.0-mm K-wire is driven through the incision in a retrograde fashion to exit at the big toe at the medial side of the nail. The wire should be midway between the dorsal and plantar aspect of the great toe. Elevation of the periosteum at the level of the neck of the fist metatarsal and osteotomy is then performed in the subcapital region of the first metatarsal with an oscillating saw perpendicular to the long axis of the shaft of the first metatarsal in the sagittal plane with slight mediolateral obliquity in the frontal plane. A small curved artery forceps is introduced in the medullary canal to displace the proximal part of the first metatarsal with derotation of the big toe to neutral position. The $\mathrm{K}$-wire is advanced proximally under vision and X-ray control into the medullary canal of the metatarsal shaft and is driven through the first tarsometatarsal joint for greater stabilization. Tourniquet release, and skin closure. The distal end of the $\mathrm{K}$ wire is curved and cut. A plantar pad is placed and an elastic bandage is taped in supination to counteract pronation of the big toe. This position is also checked weekly during follow up. 


\subsection{Post-Operative Protocol}

Patients were allowed to bear weight when tolerated. The K-wire is removed after evidence of radiological healing. Patients were then allowed to bear weight with normal shoes, and range of motion (ROM) exercises of the first metatarsophalangeal joint were carried out from then on.

\subsection{Distal Chevron Osteotomy}

The distal chevron osteotomy is done by a short medial longitudinal incision, a $60^{\circ} \mathrm{V}$-osteotomy centered on the first metatarsal head was performed and the capital fragment is displaced laterally. No soft tissue release is done in any case in our series. A below knee cast is done until healing of the osteotomy.

For all cases, radiological evaluation included radiographs on the following day, every two weeks until radiological healing and then every three months postoperatively.

\section{Results (Table 2, Figure 1)}

In the percutaneous osteotomy group (group I), the operative time ranged between 25 minutes and 40 minutes

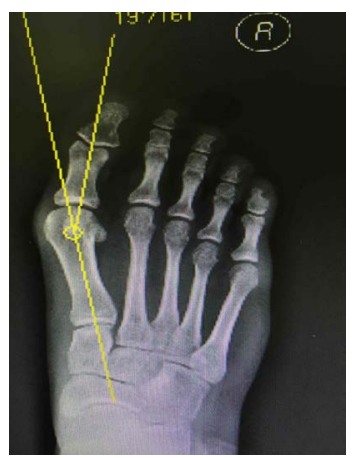

Pre-operative HV angle

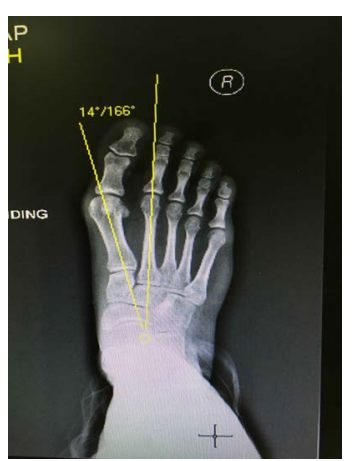

Pre-operative IM angle

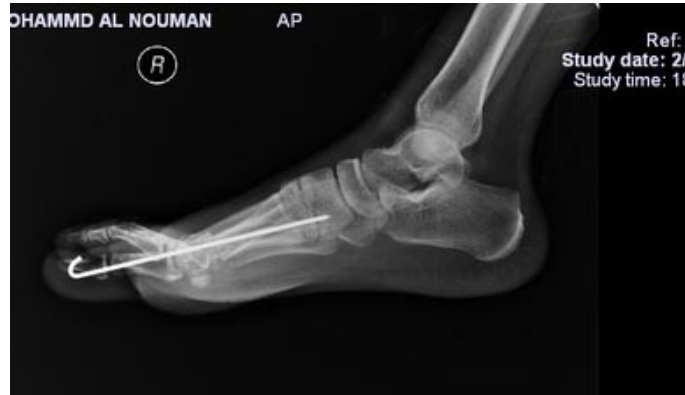

Post-operative lateral view

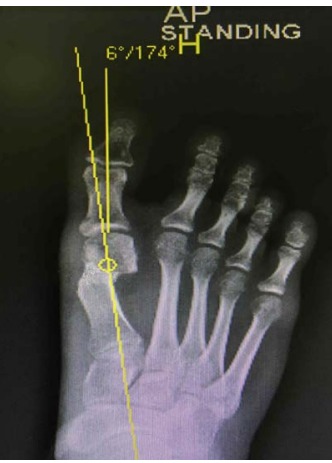

Late post-operative HV angle

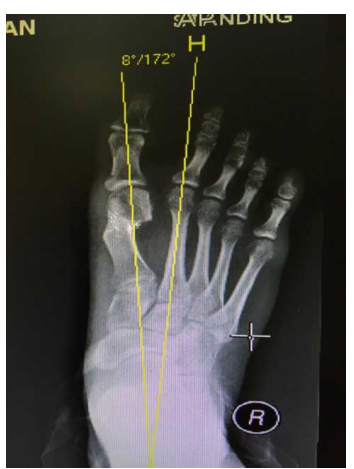

Late post-operative IM angle

Figure 1. Case Presentation of the percutaneous technique (radiological measures and follow up). 
Table 2. Post-operative data.

\begin{tabular}{cccc}
\hline & Percutaneous & Chevron & P value \\
\hline Mean operation time (minutes) & 28.81 & 39.12 & 0.001 \\
Average Follow up (Months) & 49.36 & 51.56 & 0.67849 \\
Average healing time (Weeks) & 9.3 & 9.96 & 0.56782 \\
Average shortening of M1 (mm) & 2.9 & 3.12 & 0.87567 \\
Average HV angle & 6.55 & 6.52 & 0.74532 \\
Average IM angle & 7.58 & 7.96 & 0.65735 \\
Average DMAA & 6.93 & 7.92 & 0.87865 \\
Tibial sesamoid position & 5.42 & 5.8 & 0.67743 \\
Average AOFAS & 89.6 & 86.8 & 0.87654 \\
\hline
\end{tabular}

with an average of 28.81 minutes, the follow up period ranged between 18 and 68 months with a mean 49.36 months, post-operative HV angle ranged between $6^{\circ}$ and $9^{\circ}$ with an average of $6.55^{\circ}$. The average post-operative IM angle was $7.58\left(6^{\circ}-9^{\circ}\right)$. The DMAA improved to an average of $6.93^{\circ}$ (range $6^{\circ}-9^{\circ}$ ).

The AOFAS score ranged between 80 and 94 with an average of 89.6. The tibial sesamoid position ranged between $4 \mathrm{~mm}$ and $8 \mathrm{~mm}$ with an average of $5.42 \mathrm{~mm}$. Radiological healing time ranged between 7 weeks to 14 weeks with an average of 9.3 weeks. Shortening of the first metatarsal bone ranged between 2 and $5 \mathrm{~mm}$ with an average of $2.9 \mathrm{~mm}$. As regards patient satisfaction after surgery, 22 patients were satisfied (91.66\%).

In the chevron osteotomy group, the follow up period ranged between 19 and 69 months with a mean 51.56 months, the operative time ranged between 32 minutes and 50 minutes with an average of 39.12 minutes, post-operative $\mathrm{HV}$ angle ranged between $6^{\circ}$ and $9^{\circ}$ with an average of $6.52^{\circ}$. The, the average post-operative IM angle was $7.96^{\circ}\left(7^{\circ}-9^{\circ}\right)$ The DMAA improved to an average of $7.92^{\circ}$ (range $6^{\circ}-9^{\circ}$ ). The AOFAS score ranged between 70 and 92 with an average of 86.8. The tibial sesamoid position ranged between $3 \mathrm{~mm}$ and $7 \mathrm{~mm}$ with an average of $5.8 \mathrm{~mm}$. Radiological healing time ranged between 7 weeks to 15 weeks with an average of 9.96 weeks. Shortening of the first metatarsal bone ranged between 3 and $5 \mathrm{~mm}$ with an average of $3.12 \mathrm{~mm}$. As regards patient satisfaction after surgery, 14 patients were satisfied (82.35\%).

\subsection{Complications}

In the percutaneous group, 3 feet developed pin tract infection did not necessitate removal of the $\mathrm{K}$ wire before radiological healing and were treated by appropriate antibiotics.

In the chevron osteotomy group, however, there were three major complications, the first case developed deep venous thrombosis 1 week after surgery and was treated accordingly. The second case had deep infection, which was treated by systemic antibiotics for almost 3 weeks.

Another foot showed recurrence of the deformity HV angle $>25$ degrees after chevron osteotomy and the patient refused to do any more surgery.

\subsection{Statistical Analysis}

Statistical analysis was done using SPSS software (SPSS Inc., Chicago, IL, USA). Student's t-test was used to compare demographic and pre-treatment data and a paired t-test was used to evaluate changes between the preand postoperative AOFAS, operative time, HV angle, IM angle as well as the DMAA.

In the demographic and pre-treatment data, there was no statistical difference between the two groups concerning the age, pre-operative HV angle, IM angle, or the AOFAS.

Concerning the post-operative data, in the first group, the mean correction in the HV angle was 26.69 degrees ( $\mathrm{P}=0.00001)$, the mean correction in the IM angle was 9.45 degrees $(\mathrm{P}=0.00001)$, the mean correction in the DMAA angle was $12.9^{\circ}(\mathrm{P}=0.00001)$ the mean change in the AOFAS was $47.94(\mathrm{P}=0.00001)$

In the second group, the mean change in the $\mathrm{HV}$ angle was $26.72^{\circ}(\mathrm{P}=0.0001)$, the mean change in the IM angle was $9^{\circ}(\mathrm{P}=0.00001)$, the mean correction in the DMAA angle was $11.48^{\circ}(\mathrm{P}=0.00001)$ the mean change in the AOFAS was $44.76(\mathrm{P}=0.001)$ 
Accordingly, there was significant change in the post-operative results in each group.

When comparing the results of the two groups, the only significant difference was in the operative time in favor of the percutaneous group $(\mathrm{P}=0.001)$. Otherwise, there was no significant difference in the HVA $(\mathrm{P}=$ 0.34532), IMA ( $\mathrm{P}=0.6573)$, DMAA $(0.87865)$ or in the AOFAS $(\mathrm{P}=0.87654)$

\section{Discussion}

Many operative procedures have been described for the treatment of hallux valgus including distal soft tissue procedures (DSTPs), osteotomies through the base or head of first metatarsal and proximal phalanx, arthrodesis of the metatarsocuneiform joint or metatarsophalangeal (MP) joint, excisional arthroplasty, and combined surgeries of osteotomy and DSTPs [8]-[13].

Minimally invasive procedures are supposed to correct most cases of mild to moderate hallux valgus provided that there is no arthrosis of the metatarsophalangeal joint [14].

The percutaneous technique proved to be successful for the correction of painful mild to moderate hallux valgus deformity. The clinical results achieved by many authors were comparable with those obtained by traditional open techniques. Moreover, there are many additional advantages of a minimally invasive procedure including a shorter operating time, early weight bearing, reduced risk of complications related to surgical exposure and faster recovery times. However, still there some disadvantages including long learning curve and the need for specific equipment [6] [15] [16].

Magnan et al. (2005) [16] performed 118 percutaneous distal osteotomies of the first metatarsal for painful mild-to-moderate hallux valgus in eighty-two patients. After a mean follow up of 35.9 months, the mean AOFAS was $88.2 \pm 12.9$ points. They reported significant improvement in the radiological parameters including the mean hallux valgus angle, first intermetatarsal angle, distal metatarsal articular angle, and sesamoid position. Recurrence rate was $2.5 \%$, stiffness of the first metatarsophalangeal joint was reported in $6.8 \%$, and deep infection developed in $0.8 \%$ of cases. The patients were satisfied following $91 \%$ of cases.

Enan et al., (2010) [7], treated 24 patients with mild to moderate hallux valgus by a minimally invasive approach. After a mean follow up of 21 months, the average corrections of the HV angle and the IM angle were $13^{\circ}$ and $5.4^{\circ}$ respectively.

Valles-Figueroa et al., (2010) [14] performed fifty-eight percutaneous osteotomies in 40 patients. According to the AOFAS, 28 patients (41/58 feet) had no pain. The mean functional capacity score was 41 points, and an excellent alignment was achieved in $74 \%$ of cases. The mean intermetatarsal angle was $7.5^{\circ}$.

However, some other authors reported complications after percutaneous osteotomy for hallux valgus including osteonecrosis, nonunion, malunion, and recurrence. Kadika et al. (2007) [17], performed a percutaneous distal first metatarsal osteotomy in 13 patients with mild to moderate hallux valgus. The achieved intraoperative correction was routinely lost after removal of the intramedullary Kirschner wire, leading to a high rate of recurrence of hallux valgus deformity as well as dorsal elevation of the capital fragment.

In our study, we did not report loss of correction in cases treated by percutaneous technique within the period of follow up. May be that was guaranteed by removal of $\mathrm{K}$ wires only after evidence of radiological healing in all cases.

Percutaneous distal osteotomy of the first metatarsal when performed as an isolated procedure. The results are not as good if applied for severe hallux valgus [18].

The goal of distal chevron osteotomy for hallux valgus is to restore proper first-toe joint alignment by performing lateral translation of the distal first metatarsal fragment (the metatarsal head) [19].

The chevron osteotomy, an accepted method for the correction of mild and moderate hallux valgus, is generally advocated for patients younger than the age of fifty years [20].

Vasso et al. (2015) [21] treated 184 patients with hallux valgus with a modified Austin/chevron osteotomy, after a mean follow up of 41.7 months, $93 \%$ were satisfied, the AOFAS improved from 56.6 points to 90.6, the mean HVA improved from $34.1^{\circ}$ to $6.2^{\circ}$ and the mean IM angle improved from $18.5^{\circ}$ to $4.1^{\circ}$.

Trnka et al. [18] performed chevron osteotomy in sixty-six feet. Forty-three patients (fifty-seven feet) were available for follow-up. There was only a minimal change in overall patient satisfaction, and the average score on the hallux-metatarsophalangeal-interphalangeal scale was unchanged. The passive range of motion of the first metatarsophalangeal joint decreased between the preoperative assessment and the two-year follow-up evaluation and was unchanged at the five-year follow-up evaluation. Radiographic evaluation showed no changes in the hallux valgus or intermetatarsal angle between the two-year and five-year evaluations. 
Johnson et al. (1991) [22] retrospectively compared their results with distal chevron osteotomy and a modified McBride bunionectomy. Patient satisfaction regarding pain relief was achieved in ninety-two percent of patients in the chevron group and $88 \%$ of patients in the modified McBride group.

More recently, Radwan and Mansour (2012) [23] treated 53 patients (64 feet) with mild to moderate hallux valgus by two different surgical techniques. Percutaneous distal metatarsal osteotomy was used in 33 feet. The remaining cases were treated by distal chevron osteotomy. The mean correction of HVA and IM angles $14.4^{\circ}$ and $4.8^{\circ}$ respectively, the AOFAS of the first group improved from 44.6 points to 90.2 and in the chevron group improved from 47.5 to 89.6 . At the end of follow up, $89.6 \%$ of the percutaneous group were happy for surgery whereas $64.5 \%$ in the chevron group. In our study, the comparison entails distal chevron osteotomy and a percutaneous technique with the addition of rotation of the distal fragment to neutral position which helps to correct the deformity through a short incision without too much dissection of the capsule in a relatively short operative time. Thus decreasing the risk of deep infection, post-operative stiffness and avascular necrosis.

There were many complications related to chevron osteotomy reported in the literature. The most serious complication is avascular necrosis of the first metatarsal with a reported incidence of between $0 \%$ and $20 \%$ [24] [25].

The procedure is not completely safe in older people. In our series, one case developed DVT after chevron osteotomy. She was a female fifty one years old without any previous personal history or family history of DVT before surgery.

In our series, we did not encounter that complication. However, three different major three major complications occurred in the group treated by chevron osteotomy, deep infection, DVT, and recurrence of deformity.

\section{Conclusion}

Both percutaneous osteotomy of the distal part of the first metatarsal and distal chevron osteotomy can be used to treat mild to moderate cases of hallux valgus. The outcome of both techniques is comparable concerning correction of deformity, pain reduction and foot function. However, the percutaneous technique has the advantages of shorter operative time and fewer incidences of major complications.

\section{References}

[1] Thomas, N., Joseph, M.D. and Kenneth, J.M. (2007) Decision Making in the Treatment of Hallux Valgus. Bulletin of the NYU Hospital for Joint Diseases, 65, 19-23.

[2] Sandro, G., Francesco, C., Berto, B. and Francesca, V. (2003) Hallux Valgus Surgery: The Minimally Invasive Bunion Correction (SERI). Techniques in Foot and Ankle Surgery, 2, 11-12.

[3] Roddy, E., Zhang, W. and Doherty, M. (2008) Prevalence and Associations of Hallux Valgus in a Primary Care Population. Arthritis Care \& Research, 59, 857-862. http://dx.doi.org/10.1002/art.23709

[4] Leemrijse, T., Valtin, B. and Besse, J.L. (2007) Hallux Valgus Surgery in 2005. Conventional, Mini-Invasive or Percutaneous Surgery? Uni- or Bilateral? Hospitalization or One-Day Surgery? Revue de Chirurgie Orthopédique et Réparatrice de l'Appareil Moteur, 94, 111-127.

[5] Schuh, R., Willegger, M., Holinka, J. and Wanivenhaus, A.H. (2013) Angular Correction and Complications of Proximal First Metatarsal Osteotomies for Hallux Valgus Deformity. International Orthopaedics, 37, 1771-1780. http://dx.doi.org/10.1007/s00264-013-2012-4

[6] Botezatu, I., Marinescu, R. and Laptoiu, D. (2015) Minimally Invasive-Percutaneous Surgery-Recent Developments of the Foot Surgery Techniques. Journal of Medicine and Life, 8, 87-93.

[7] Enan, A., Abo-Hegy, M. and Seife, H. (2010) Early Results of Distal Metatarsal Osteotomy through Minimally Invasive Approach for Mild-to-Moderate Hallux Valgus. Acta Orthopredica Belgica, 76, 526-535.

[8] Bonney, G. and Macnab, I. (1952) Hallux Valgus and Hallux Rigidus. A Critical Survey of Operative Results. The Journal of Bone \& Joint Surgery (Br), 34, 366-385.

[9] McBride, E.D. (1967) The McBride Bunion Hallux Valgus Operation. Refinements in the Successive Surgical Steps of the Operation. The Journal of Bone \& Joint Surgery (Am), 49, 1675-1683.

[10] Erturer, E., Aksoy, B., Beki, S. and Ozturk, I. (2004) Radiographic and Functional Results of the Lindgren-Turan Operation in the Treatment of Hallux Valgus. Acta Orthopaedica et Traumatologica Turcica, 38, 125-129.

[11] Faber, F.W.M., Mulder, P.G.H. and Verhaar, J. (2004) Role of First Ray Hypermobility in the Outcome of the Hohmann and the Lapidus Procedure. A Prospective, Randomized Trial Involving One Hundred and One Feet. The Journal of Bone \& Joint Surgery (Am), 86, 486-495. 
[12] Schneider, W., Aigner, N., Pinggera, O. and Knahr, K. (2004) Chevron Osteotomy in Hallux Valgus. Ten-Year Results of 112 Cases. The Journal of Bone \& Joint Surgery (Br), 86, 1016-1020 http://dx.doi.org/10.1302/0301-620X.86B7.15108

[13] Yamamoto, K., Imakiire, A., Katori, Y. and Koizumi, R. (2005) Clinical Results of Modified Mitchell’s Osteotomy for Hallux Valgus Augmented with Oblique Lesser Metatarsal Osteotomy. Journal of Orthopaedic Surgery (Hong Kong), 13, 245-252.

[14] Valles-Figueroa, J.F., Rodríguez-Reséndiz, F., Caleti-del Mazo, E., Malacara-Becerra, M. and Suárez-Ahedo, C.E. (2010) Percutaneous Distal Metatarsal Osteotomy for the Correction of Hallux Valgus. Acta Ortopédica Mexicana, 24, 385-389.

[15] Bösch, P., Markowski, H. and Rannicher, V. (1990) Technique and Initial Results of Subcutaneous Distal Metatarsal Osteotomy. Orthopädische Praxis, 26, 51-56.

[16] Magnan, B., Pezzè, L., Rossi, N. and Bartolozzi, P. (2005) Percutaneous Distal Metatarsal Osteotomy for Correction of Hallux Valgus. The Journal of Bone \& Joint Surgery (Am), 87, 1191-1199. http://dx.doi.org/10.2106/jbjs.d.02280

[17] Kadakia, A.R. Smerek, J.P. and Myerson, M.S. (2007) Radiographic Results after Percutaneous Distal Metatarsal Osteotomy for Correction of Hallux Valgus Deformity. Foot \& Ankle International, 28, 355-360. http://dx.doi.org/10.3113/FAI.2007.0355

[18] Díaz-Fernández, R. (2015) Treatment of Moderate and Severe Hallux Valgus by Performing Percutaneous Double Osteotomy of the First Metatarsal Bone. Revista Española de Cirugía Ortopédica y Traumatología (English Edition), 59, 52-58.

[19] Akpinar, E., Buyuk, A.F., Cetinkaya, E., Gursu, S., Ucpunar H, and Albayrak A. (2016) Proximal Intermetatarsal Divergence in Distal Chevron Osteotomy for Hallux Valgus: An Overlooked Finding. The Journal of Foot \& Ankle Surgery, 55, 504-508. http://dx.doi.org/10.1053/j.jfas.2016.01.043

[20] Trnka, H.J., Zembsch, A., Easley, M.E., Salzer, M., Ritschl, P. and Myerson, M.S. (2000) The Chevron Osteotomy for Correction of Hallux Valgus. Comparison of Findings after Two and Five Years of Follow-Up. The Journal of Bone \& Joint Surgery (Am), 82-A, 1373-1378.

[21] Vasso, M., Del Regno, C., D'Amelio, A. and Schiavone Panni, A. (2016) A Modified Austin/Chevron Osteotomy for Treatment of Hallux Valgus and Hallux Rigidus. Journal of Orthopaedics and Traumatology, 17, 89-93.

[22] Johnson, J.E., Clanton, T.O., Baxter, D.E. and Gottlieb, M.S. (1991) Comparison of Chevron Osteotomy and Modified McBride Bunionectomy for Correction of Mild to Moderate Hallux Valgus Deformity. Foot \& Ankle International, 12, 61-68. http://dx.doi.org/10.1177/107110079101200201

[23] Radwan, Y.A. and Mansour, A.M. (2012) Percutaneous Distal Metatarsal Osteotomy versus Distal Chevron Osteotomy for Correction of Mild-to-Moderate Hallux Valgus Deformity. Archives of Orthopaedic and Trauma Surgery, 132, 1539-1546. http://dx.doi.org/10.1007/s00402-012-1585-5

[24] Green, M.A., Dorris, M.F., Baessler, T.P., Mandel, L.M. and Nachlas, M.J. (1993) Avascular Necrosis Following Distal Chevron Osteotomy of the First Metatarsal. Journal Foot and Ankle Surgery, 32, 617-622.

[25] Donnelly, R.E., Saltzman, C.L., Kile, T.A. and Johnson, K.A. (1994) Modified Chevron Osteotomy for Hallux Valgus. Foot \& Ankle International, 15, 642-645. http://dx.doi.org/10.1177/107110079401501202 